\title{
A NOTE ON IDEALS IN THE DISC ALGEBRA
}

\author{
FRANK FORELLI ${ }^{1}$
}

\begin{abstract}
We offer an elementary theorem on ideals in the disc algebra $A(D)$, which by way of a corollary, one, identifies the maximal ideals of $A(D)$, and two, provides a proof, which avoids the axiom of choice, that every proper ideal in $A(D)$ is contained in a maximal ideal.
\end{abstract}

1. Let $A$ be a ring of functions in the closed unit disc $\overline{\mathbf{D}}$, each of which is continuous in $\overline{\mathbf{D}}$ and holomorphic in $\mathbf{D}$. (Thus $A \leqslant A(\mathbf{D})$.) Suppose that

(a) $A$ is dense in the disc algebra $A(D)$, i.e. if $f \in A(D)$ and $\varepsilon>0$, then there is a $g$ in $A$ with $|f-g|<\varepsilon$ in D.

Then we have the following.

THEOREM. Let $Q$ be an ideal in $A, Q \neq 0$, and let

$$
X \subset \bigcup_{f \in Q}\{f \neq 0\}
$$

where by $\{f \neq 0\}$ we mean the set of those $z$ in $\overline{\mathbf{D}}$ for which $f(z) \neq 0$. If $X$ is closed in $\overline{\mathbf{D}}$, or if $A=A(\mathbf{D})$ and

$$
X \cap \mathbf{T}=\bar{X} \cap \mathbf{T}
$$

where by $\bar{X}$ we mean the closure of $X$ in $\overline{\mathbf{D}}$, then there is an $f$ in $Q$ with $f$ vanishing nowhere in $X$.

We will come to the proof in due course. The theorem raises the following question (that we are unable to answer). Which ideals $Q$ in $A, Q \neq 0$, have the property that if $X$ is equal to the right side of (1), then there is an $f$ in $Q$ with $f$ vanishing nowhere in $X$ ? This holds if $Q$ is principal. What if $Q$ is finitely generated?

Does it hold if $Q$ is an ideal of denominators, i.e. if

$$
Q=Q(\gamma)=\{f: f \in A, f \gamma \in A\}
$$

where $\gamma$ is in the field of fractions $A_{(0)}$ of $A$ ? If yes, this would imply (see the proof of the corollary below) that if $X$ is any subset of $\overline{\mathbf{D}}, X \neq \varnothing$, then

$$
A_{X}=\bigcap_{\zeta \in X} A_{\zeta}
$$

where by $A_{X}$ we mean the ring of fractions

$$
\{g / f: g, f \in A, f \text { vanishes nowhere in } X\},
$$

Received by the editors March 30, 1981.

1980 Mathematics Subject Classification. Primary 30H05.

'Supported by the National Science Foundation. 
and by $A_{\zeta}, \zeta \in \overline{\mathbf{D}}$, we mean the local ring of fractions

$$
\{g / f: g, f \in A, f(\zeta) \neq 0\} .
$$

If not, then one might ask for which $X \subset \overline{\mathbf{D}}$ does (4) hold. In this regard we have the following.

Corollary. Let $X \subset \overline{\mathbf{D}}, X \neq \varnothing$. If $X$ is closed in $\overline{\mathbf{D}}$, or if $A=A(\mathbf{D})$ and (2) holds, then (4) holds.

Proof. Let $\gamma$ belong to the right side of (4), and put $Q=$ the right side of (3). Then (1) holds; hence there is an $f$ in $Q$ with $f$ vanishing nowhere in $X$. This proves that $\gamma \in A_{X}$.

Suppose that in addition to (a) we have

(b) If $f \in A$, and $f$ vanishes nowhere in $\overline{\mathbf{D}}$, then $1 / f \in A$.

Then the following holds.

Corollary. Let $Q$ be an ideal in $A, Q \neq A$. Then there is a $\zeta$ in $\overline{\mathbf{D}}$ such that $Q \subset P_{\zeta}$ where

$$
P_{\zeta}=\{f: f \in A, f(\zeta)=0\}
$$

Proof. Otherwise, in the statement of the theorem we may take $X=\overline{\mathbf{D}}$.

Our proof of the theorem is very elementary; its main ingredient is that if

$$
\varphi=\sum_{-k}^{k} \alpha_{j} e^{i j \theta}
$$

is a trigonometric polynomial, then

$$
e^{i k \theta} \varphi=g \mid \mathbf{T}
$$

where $g \in A(\mathrm{D})$.

By the second corollary, every maximal ideal in $A$ is a $P_{\zeta}, \zeta \in \overline{\mathbf{D}}$; on the other hand, if $A$ is an algebra over $\mathbf{C}$, then every $P_{\zeta}$ is maximal. This identifies the maximal ideals of $A(D)$ in a way which is more elementary than those in [3]. To turn the second corollary around, we have an easy and elementary proof (which does not use the axiom of choice) of the fact that if $f_{1}, \ldots, f_{n}$ in $A$ do not have a common zero in $\overline{\mathbf{D}}$, then $\left(f_{1}, \ldots, f_{n}\right)=(1)$. Other proofs of this (if $\left.A=A(\mathbf{D})\right)$ which avoid the axiom of choice are in [1] and [2].

We might point out that Theorem 1 is to some extent peculiar to the disc D. For example, it fails for the ball algebra $A(\mathbf{B})$ in 2 variables (let $Q=P_{0}=\{f: f \in A(\mathbf{B})$, $\left.f(0)=0\}, X=\partial \mathbf{B}=\left\{(z, w):|z|^{2}+|w|^{2}=1\right\}\right)$.

2. We now come to the proof of the theorem. To begin, let $A=A(D)$. Since $X \cap \mathrm{T}$ is compact, we have

$$
X \cap \mathbf{T} \subset \bigcup_{j=1}^{n}\left\{f_{j} \neq 0\right\}
$$


where $f_{1}, \ldots, f_{n} \in Q$. Let $Y=X \cap \mathbf{T}$. WLOG we may assume that $Y \neq \varnothing$. Put

$$
f=\sum_{j=1}^{n} \bar{f}_{j} f_{j}
$$

let

$$
\alpha=\inf _{Y} f, \quad \beta=\inf _{\mathbf{T}}\left(1 / \sum_{j=1}^{n}\left|f_{j}\right|\right),
$$

and choose trigonometric polynomials $\varphi_{1}, \ldots, \varphi_{n}$ such that $\left|\bar{f}_{j}-\varphi_{j}\right|<\alpha \beta$. Then (on T)

$$
\left|f-\sum_{j=1}^{n} \varphi_{j} f_{j}\right|=\left|\sum_{j=1}^{n}\left(\bar{f}_{j}-\varphi_{j}\right) f_{j}\right|<\alpha,
$$

hence $\Sigma \varphi_{j} f_{j}$ vanishes nowhere in $Y$. Replacing $\varphi_{j}$ by $e^{i k \theta} \varphi_{j}$ (where $k \geqslant \operatorname{deg} \varphi_{j}$, $1 \leqslant j \leqslant n$ ), we obtain (cf. (5)) $g_{1}, \ldots, g_{n}$ in $A$ (D) with $\Sigma g_{j} f_{j}$ vanishing nowhere in $Y$. Let

$$
g=\sum_{j=1}^{n} g_{j} f_{j}
$$

Then $g \in\left(f_{1}, \ldots, f_{n}\right)$. Since $g$ vanishes nowhere in $\bar{X} \cap \mathbf{T}, g$ has at most a finite number of zeros in $X$ (counting multiplicities), say $\zeta_{1}, \ldots, \zeta_{m}$. We have $\zeta_{j} \in \mathbf{D}$, hence

$$
g(z)=\left(z-\zeta_{1}\right) \cdots\left(z-\zeta_{m}\right) h(z)
$$

where $h \in A(\mathbf{D}), h$ vanishes nowhere in $X$.

Let $1 \leqslant j \leqslant m$, and choose $h_{j}$ in $Q$ with $h_{j}\left(\zeta_{j}\right) \neq 0$. We have

$$
h_{j}(z)-h_{j}\left(\zeta_{j}\right)=\left(z-\zeta_{j}\right) \psi_{j}(z)
$$

where $\psi_{j} \in A(\mathbf{D})$, i.e.

$$
h_{j}\left(\zeta_{j}\right)=h_{j}-\left(z-\zeta_{j}\right) \psi_{j}
$$

Put

$$
\mu=\prod_{j=1}^{m} h_{j}\left(\zeta_{j}\right)
$$

then (6) and (7) give

$$
\mu h \in\left(f_{1}, \ldots, f_{n}, h_{1}, \ldots, h_{m}\right) .
$$

Thus $h \in Q$, which completes the proof of the theorem if $A=A(\mathbf{D})$.

If $A \neq A(\mathbf{D})$, put

$$
P=\left\{\sum_{1}^{k} f_{j} g_{j}: f_{j} \in A(\mathbf{D}), g_{j} \in Q\right\} .
$$

Then $P$ is an ideal in $A(\mathbf{D})$, with $Q \subset P$; by the foregoing there is an $f$ in $P$ with $f$ vanishing nowhere in $X$. We have

$$
f=\sum_{j=1}^{n} f_{j} g_{j}
$$


where $f_{j} \in A(\mathbf{D}), g_{j} \in Q$. Let

$$
\alpha=\inf _{X}|f|, \quad \beta=\inf _{\mathbf{D}}\left(1 / \sum_{j=1}^{n}\left|g_{j}\right|\right) .
$$

By the hypothesis (a) we may choose $h_{j}$ in $A, 1 \leqslant j \leqslant n$, with $\left|f_{j}-h_{j}\right|<\alpha \beta$. Put

$$
h=\sum_{j=1}^{n} h_{j} g_{j} \text {. }
$$

Then $h \in Q$; and

$$
|f-h|=\left|\sum_{j=1}^{n}\left(f_{j}-h_{j}\right) g_{j}\right|<\alpha,
$$

hence $h$ vanishes nowhere in $X$.

3. In the foregoing we may replace $\overline{\mathbf{D}}$ by any bordered Riemann surface $\bar{W}$ which is compact. Our proof of the theorem (and its corollaries) would then work for any ring of functions in $A(W)$ which is dense in $A(W)$. One should replace the trigonometric polynomials on $\mathbf{T}$ by the separating selfadjoint algebra in $C(\partial W)$ consisting of the complex linear span of quotients of inner functions in $A(W)$ (cf. [4], where it is proved that the inner functions in $A(W)$ separate points in $\partial W)$.

\section{REFERENCES}

1. L. Carleson, On bounded analytic functions and closure problems, Ark. Math. 2 (1952), 283-291.

2. P. J. Cohen, A note on constructive methods in Banach algebras, Proc. Amer. Math. Soc. 12 (1961), $159-163$.

3. K. Hoffman, Banach spaces of analytic functions, Prentice-Hall, Englewood Cliffs, N. J., 1962.

4. E. L. Stout, On some algebras of analytic functions on finite open Riemann surfaces, Math. Z. 92 (1966), 366-379.

DePaRTMENT OF MATHEMatics, University OF Wisconsin, Madison, Wisconsin 53706 